\title{
Economic evaluation of genetic screening for Lynch syndrome in Germany
}

\author{
Franziska Severin, MPH${ }^{1}$, Björn Stollenwerk, PhD¹, Elke Holinski-Feder, MD², Elisabeth Meyer, PhD', \\ Volker Heinemann, MD, PhD³, Clemens Giessen-Jung, MD³ and Wolf Rogowski, PhD'
}

\begin{abstract}
Purpose: Lynch syndrome (LS) screening among patients with newly diagnosed colorectal cancer can decrease mortality in their affected first-degree relatives. In Germany, it is not yet clinical practice and the cost-effectiveness of different testing strategies is unknown.

Methods: We developed a decision-analytic model to analyze the cost-effectiveness of LS screening from the perspective of the German Statutory Health Insurance system. A total of 22 testing strategies considering family-history assessment, analysis of tumor samples (i.e., immunohistochemistry (IHC), microsatellite instability, and $B R A F$ mutation testing) and genetic sequencing were analyzed. Life-years gained in relatives by closedmeshed colonoscopy and aspirin prophylaxis were estimated by Markov models. Uncertainty was assessed deterministically and probabilistically.
\end{abstract}

Results: On average, detected mutation carriers gained 0.52 lifeyears (undiscounted: 1.34) by increased prevention. Most strategies were dominated, with three exceptions: family assessment by the Bethesda criteria followed by IHC and BRAF testing and genetic sequencing; IHC and $B R A F$ testing and genetic sequencing; and direct sequencing of all index patients. Their incremental cost-effectiveness was $€ 77,268, € 253,258$, and $€ 4,188,036$ per life-year gained, respectively.

Conclusion: The results were less favorable than those of previous models. Chemoprevention appears to provide comparably low additional benefit and improves cost-effectiveness only slightly.

Genet Med advance online publication 8 January 2015

Key Words: cost-effectiveness; Germany; individualized healthcare; Lynch syndrome; screening

\section{INTRODUCTION}

In Germany, colorectal cancer (CRC) is the second most commonly diagnosed form of cancer for both women and men, with more than 69,000 cases reported in 2012 (ref. 1). Lynch syndrome (LS), also known as hereditary nonpolyposis colorectal cancer, accounts for $\sim 3 \%$ of all newly diagnosed CRC cases, which makes it the most common hereditary colon cancer syndrome. ${ }^{2}$ It is caused by autosomal dominant mutations in DNA mismatch-repair (MMR) genes (MSH2, MLH1, MSH6, and PMS2), which lead to an accumulation of genetic changes, furthering tumor growth. ${ }^{3}$ Individuals who inherit the autosomal dominant mutation have an $\sim 40 \%$ lifetime risk of developing CRC, ${ }^{4}$ as compared with $~ 6 \%$ in the German population. ${ }^{1}$

In Germany, patients with LS are recommended to undergo colonoscopy every year from age 25 , along with physical examinations. ${ }^{5,6}$ Colonoscopic surveillance has been shown to be effective at preventing progression (of polyps) to CRC as well as at detecting the disease at an early stage, thus reducing CRC incidence and mortality. ${ }^{7}$ In addition to increased colonoscopic surveillance, long-term aspirin intake has been proposed as an effective way to reduce CRC risks. ${ }^{8}$ Although aspirin prophylaxis is internationally discussed as a promising approach, it is not generally recommended by the German guidelines for the prevention of CRC in LS patients because data regarding appropriate dosage are still missing. ${ }^{5}$

Given the high CRC risk in LS carriers, it is important to define appropriate diagnostic procedures for the identification of LS patients. Traditionally, clinical criteria such as the revised Bethesda guidelines or Amsterdam II criteria have been used to guide further genetic testing in patients suspected of having LS. ${ }^{5}$ These criteria include information about cancer cases among family members. However, many patients might not be identified by family criteria because of small family sizes, incomplete penetrance of the MMR mutation, or patients simply being unaware of cancer cases in their families. ${ }^{9}$ Therefore, alternative screening algorithms that do not include familyhistory data might be desirable. ${ }^{10,11}$

International studies have shown that genetic screening among patients with newly diagnosed CRC to detect mutations and offer testing and increased prevention to relatives may be an effective approach, resulting in very favorable cost-effectiveness ratios of less than $\$ 40,000$ per life-year gained (LYG) ${ }^{12-17}$ or less than $\$ 60,000$ per quality-adjusted LYG. ${ }^{18,19}$ We would expect similar favorable results for Germany; however, given international differences in medical reimbursement rates and health-care provision, this still needs to be assessed. In particular, the German

\footnotetext{
${ }^{1}$ Institute for Health Economics and Health Care Management, Helmholtz Zentrum München, German Research Center for Environmental Health (GmbH), Neuherberg, Germany ${ }^{2}$ Medizinische Klinik und Poliklinik IV, Campus Innenstadt, Klinikum der Universität München, Munich, Germany; ${ }^{3}$ Department of Medical Oncology and Comprehensive Cancer Center, Ludwig-Maximilians-Universität München, Munich, Germany. Correspondence: Franziska Severin (franziska.severin@helmholtz-muenchen.de)
} 
guidelines foresee yearly colonoscopic surveillance, whereas existing cost-effectiveness models assume surveillance intervals of 2 or even 3 years. ${ }^{12,14-17}$ Also, costs of testing for LS appear to be higher in Germany than those reported in US models. ${ }^{13-16,18,19}$ Both might possibly increase the costs per LYG of LS screening in Germany.

To our knowledge there is currently only one study assessing the cost-effectiveness of LS screening in Germany. ${ }^{20}$ Although this study concludes that the most cost-effective approach involves testing first-degree relatives (FDRs) of newly diagnosed CRC cases who have been found to be mutation carriers, ${ }^{20}$ it does not compare different testing strategies to detect LS in CRC patients.

Therefore, this study aims to assess the expected cost-effectiveness of different screening strategies for LS from the perspective of the German Statutory Health Insurance system. The Statutory Health Insurance system is the most important payer in the German health-care system, with $\sim 85 \%$ of the German population seeking insurance within the system. ${ }^{21}$ We also aimed to assess the cost-effectiveness of adding aspirin chemoprevention to colonoscopic surveillance in LS carriers. Existing studies do not account for the presumably favorable effect of aspirin chemoprevention in LS carriers, and thus the potential role of aspirin in LS remains unclear. Moreover, we aimed to identify key variables that could improve the cost-effectiveness of LS screening and that thus should be further considered by German public policy.

\section{MATERIALS AND METHODS}

\section{Model structure}

We set up a probabilistic decision-analytic model analyzing effects in LYG and costs financed by the Statutory Health Insurance. We used a cohort model consisting of two parts. First, the genetic testing strategies were represented as a decision tree. This allowed evaluation of various testing strategies in terms of the number of detected mutations and related costs. Second, Markov models were used to estimate cancer-related mortality and life expectancy in FDRs without LS, in undetected FDRs who inherited LS but who undergo normal colonoscopic surveillance only, and in FDRs who had LS detected and thus can benefit from increased surveillance and prophylactic aspirin. To illustrate the consequences for the Statutory Health Insurance, we assume a cohort size of 69,400 individuals with CRC diagnosed, which corresponds to the CRC incidence projected for Germany in 2012 (ref. 1).

Testing strategies. Once CRC is identified in an index patient, genetic counseling and testing for LS are offered. In those patients who agree to be tested, analysis of tumor material and genetic sequencing are performed. Genetic sequencing involves testing of a blood sample to identify mutations in the MMR genes by means of sequencing and deletion. Tumor screening involves microsatellite instability (MSI) and immunohistochemistry (IHC) analysis and testing for the BRAF V600 mutation. In contrast to MSI testing, IHC can predict which of the MMR genes may be defective, resulting in lower testing costs because genetic sequencing can be targeted to the relevant MMR gene. If the MLH1 protein is absent in the IHC analysis, BRAF testing can be used as a further step to exclude sporadic CRC cases: detection of the BRAF V600 mutation strongly argues for a sporadic tumor.

Figure 1 provides an overview of the screening strategies considered. In strategies 1-6, tumor tissue analysis is used as a preceding step to increase the likelihood of identifying a germ-line mutation in an MMR gene. Strategy 7 assumes direct sequencing of all MMR genes without prior tumor analysis. The seven baseline strategies may be combined with the Amsterdam $\mathrm{II}^{22}$ (A) or revised Bethes $\mathrm{da}^{23}$ (B) criteria. Testing is then offered only to patients who fulfill the clinical criteria. The resulting testing strategies are termed A 1-7 and B 1-7. Strategies B-4 and B-6 are the two screening strategies currently recommended by the German guidelines for CRC treatment and prevention. ${ }^{5}$

If a causative mutation is detected in an index patient, targeted DNA testing for this mutation can be offered to the person's FDRs. FDRs who inherited the family mutation are offered increased prevention opportunities.

Markov model. Markov models were used to estimate cancerrelated mortality and life expectancy in FDRs. Following the recommendation of the German guidelines for CRC, ${ }^{5}$ LSpositive FDRs were assumed to be offered annual colonoscopic surveillance starting at age 25 . In addition, although not yet recommended by the German guidelines, they receive aspirin prophylaxis. Mutation-negative relatives are offered standard prevention consisting of colonoscopy every 10 years between ages 55 and 75. Standard prevention would also be offered to false-negative mutation carriers and those with unknown mutation status (e.g., relatives who decline genetic testing).

Each Markov model consists of the five main states: well, CRC, metachronous CRC, well after cancer, and death (Figure 2). Cancer stages were classified according to the Union Internationale Contre le Cancer stages I-IV, and survival was assumed to depend on cancer stage at tumor detection. Evidence suggests that the stage distribution is more favorable in individuals with intensified screening and that colonoscopic surveillance is effective by detecting the disease at an early stage. ${ }^{6}$

Once CRC is diagnosed, FDR could progress to metachronous cancer state. We assumed that an individual could develop CRC not more than twice in his or her lifetime. ${ }^{13,14}$ For both initial and metachronous cancer, FDRs progressed to the state of well after cancer if they had survived CRC for more than 10 years.

The model used 1-year cycle length to capture short-term and long-term costs and effects associated with CRC. The Markov process continued until the age of 120 years, assuming that virtually all cohort members will have died by this age.

Analysis was performed using TreeAge Pro 2014 (TreeAge Software, Williamstown, MA) with a discount rate of 3\%. Halfcycle correction was applied to both costs and effects. 


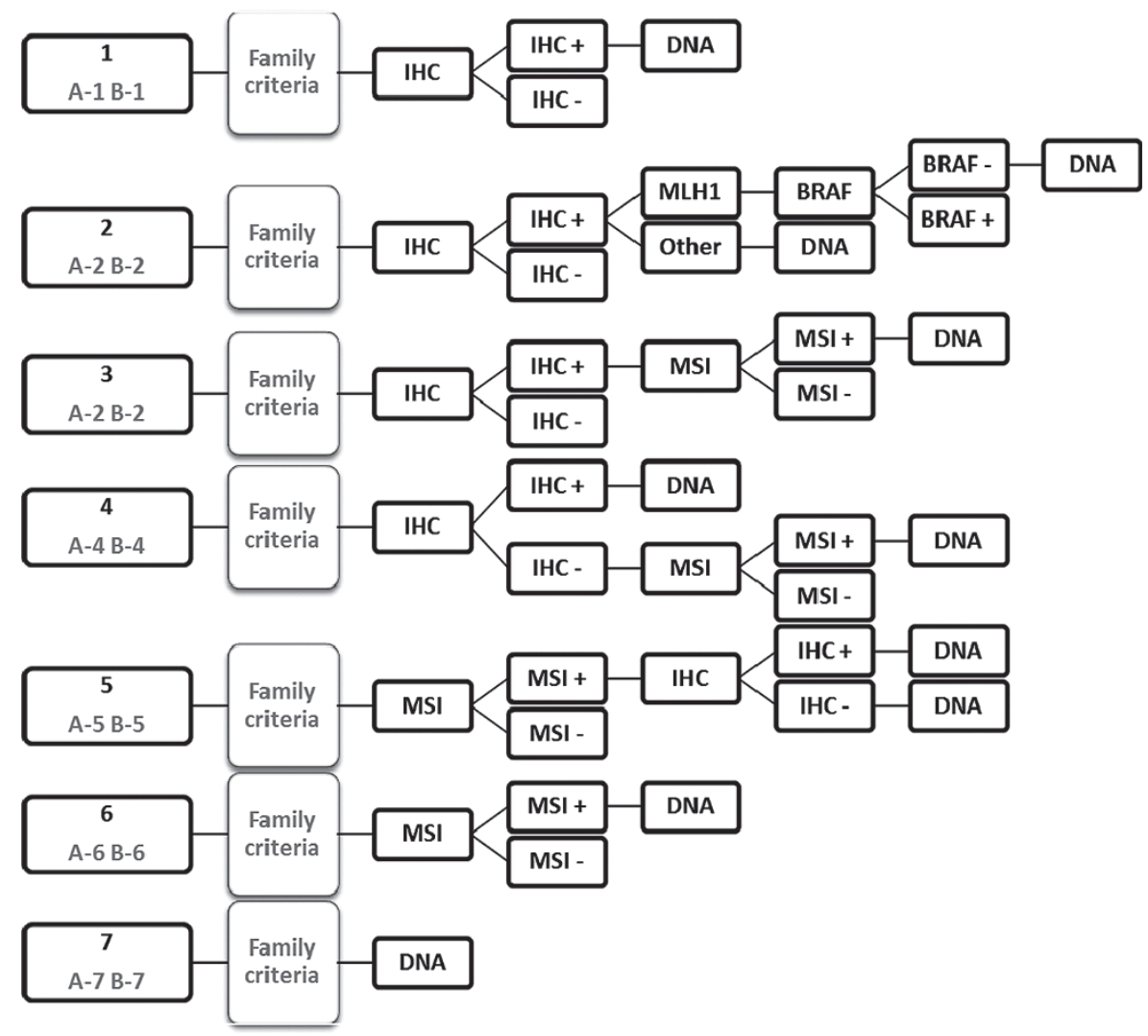

Figure 1 Overview of screening strategies. Strategy 1 starts with IHC testing, followed by genetic sequencing if results were abnormal. Strategy 2 in addition involves testing for the BRAF V600E mutation as an intermediate step in case the MLH1 protein is absent. If this mutation is found in an MLH1-absent tumor, the cancer is most likely sporadic, and further testing is omitted. Strategy 3 involves sequential IHC and MSI analysis, which must both have a positive result to trigger genetic testing. In contrast to this, Strategy 4 foresees MSI testing in case of a negative IHC test result, and genetic testing is performed if indicated by either method. Strategy 5 starts with MSI testing, followed by both IHC testing and genetic sequencing in case of a positive MSI result. Strategy 6 comprises MSI testing only. Strategy 7 assumes direct sequencing of the MMR genes. All seven baseline strategies may be combined with the Amsterdam II (A) or revised Bethesda (B) criteria. Testing is then offered only to patients who fulfill the clinical criteria. The resulting testing strategies are termed A 1-7 and B 1-7. BRAF, BRAF V 600E mutation testing; DNA, genetic sequencing to identify mutations in the MMR genes; IHC, immunohistochemistry; MSI, microsatellite instability.

\section{Parameters}

We obtained input parameters (see Supplementary Material S1 online for an overview) from the published literature using PubMed searches and reference tracking.

Family information and test uptake. Data regarding the number of FDRs per index patient and relatives' sex and age were obtained from German studies. ${ }^{6,24,25}$ Family mutation prevalence among FDRs was assumed to be $50 \%$, reflecting the autosomal dominant inheritance. Data regarding the MMR genes affected were taken from a metaanalysis. ${ }^{2}$

The uptake of genetic testing among index patients was assumed to be $85 \%$, as reported by Dutch study. ${ }^{26}$ Uptake of genetic testing among FDRs was estimated to be $29.5 \%$, as reported by a German study. ${ }^{25}$ Based on a meta-analysis of international studies, adherence to regular colonoscopy among mutation-positive relatives was assumed to be $82 \% .^{2}$ Patients who adhere to colonoscopic prevention are also assumed to comply with aspirin prevention.

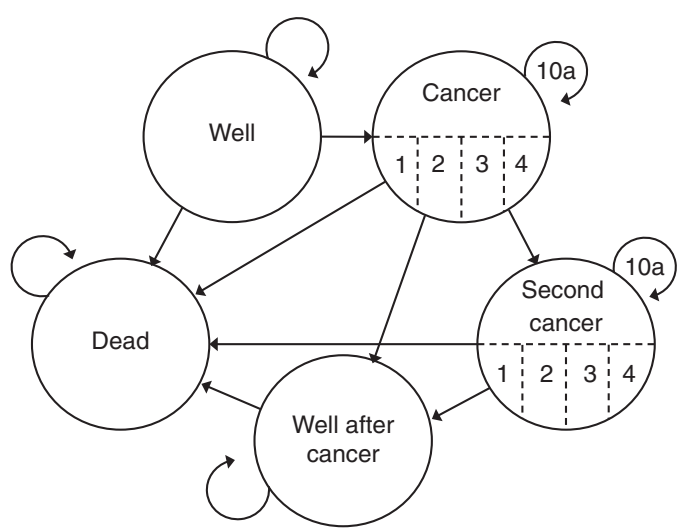

Figure 2 Markov model. Markov models were used to estimate CRCrelated mortality and costs of CRC treatment and prevention. The major clinical events were no cases of CRC (state Well), the development of an initial case of CRC (state Cancer), the development of a metachronous case of CRC (state Second cancer), survival of a first or metachronous case of CRC for more than 10 years (state Well after cancer) and death from CRC or other causes (state Dead). CRC was classified according to the UICC (Union Internationale Contre le Cancer) stages I-IV. CRC, colorectal cancer. 
Test quality. Sensitivity and specificity of laboratory tests were taken from a systematic review. ${ }^{2}$ Performance of clinical criteria was taken from a recent international study. ${ }^{27}$ The family mutation test in relatives was assumed to have $100 \%$ sensitivity and specificity. ${ }^{14}$

Disease epidemiology. Data regarding the risk of CRC for mutation carriers were taken from a recent French study. ${ }^{4}$ The cumulative risk was estimated at $42 \%$ by age 80 years, averaged over different MMR genes. This number is comparable with a recent consensus estimate. ${ }^{2}$ The cumulative risks for the different age categories reported in the study were converted into 1 -year probabilities via conversion to rate. ${ }^{28}$ Cumulative CRC risks for the general population were obtained from the Centre for Cancer Registry Data at Robert-Koch-Institute ${ }^{29}$ and were also transferred to 1-year risks.

Survival rates by Union Internationale Contre le Cancer stage and year after cancer diagnosis were taken from the Munich Tumor Registry. As compared with sporadic CRC, MSI-associated tumors are characterized by better survival. Following the evidence generated by Popat et al., ${ }^{30}$ the model assumes a relative risk of $67 \%$ for overall survival associated with MSI.

Effectiveness of screening. Data regarding the distribution of CRC stages in the general population were obtained from the Munich Cancer Registry. The stage distribution for LS carriers, with and without increased prevention, was taken from a German study in LS patients. ${ }^{6}$

The study by Stupart et al. ${ }^{7}$ provides an odds ratio of colon cancer incidence for patients with LS who undergo regular colonoscopy, as compared with nonadherent mutation carriers. This odds ratio corresponds with a median follow-up time of 5 years in a population with an average age of 34 years. Based on the exponential distribution, this corresponds with an average follow-up time of 7.21 years. To convert this odds ratio to a hazard rate, we applied the incidence rates of Bonadona et al. ${ }^{4}$ because the colon cancer incidence has not been reported by Stupart et al.

Recent data from the CAPP2 study showed that daily aspirin use $(600 \mathrm{mg})$ for 4 years had a protective effect on CRC in LS patients. ${ }^{8}$ In accordance with this study, our model assumes a $63 \%$ hazard ratio of CRC for a maximum of 11 years, which corresponds to the observation period of the study.
Costs. Costs are calculated in 2012 euros $(€)$. Costs for the genetic testing process and colonoscopic prevention were calculated from the German ambulatory fee schedule, based on fixed points per type of service multiplied by a baseline point value. Costs for aspirin are based on data reported by Bayer HealthCare.

To estimate the costs of complications from colonoscopy and aspirin prevention, International Classification of Diseases codes for the main adverse events, bleeding and perforation (as a complication of colonoscopy) and nontrivial gastrointestinal bleeding (as complication of aspirin prevention), were identified. From the German Diagnosis Related Groups Report Browser (Version 2012), ${ }^{31}$ the most common Diagnosis Related Groups for these International Classification of Diseases codes were extracted. The average case costs for these Diagnosis Related Groups were taken as a proxy for the costs of hospitalization and were multiplied with the probability of being hospitalized after an adverse event. Potential ambulatory costs were considered negligible.

Costs for treating CRC at different Union Internationale Contre le Cancer stages were taken from Mvundura et al. ${ }^{14}$ and transferred based on purchasing power parities data provided by the Organisation for Economic Co-operation and Development.

\section{Uncertainty}

Probabilistic. Input parameters were assigned statistical distributions by fitting beta distributions for probabilities, gamma distributions for cost, Dirichlet distributions for multinomial data, and log-normal distributions for parameters associated with risk reductions. A probabilistic sensitivity analysis for the nondominated strategies with 1,000 iterations was conducted.

Decision uncertainty is presented by means of cost-effectiveness acceptability curves. In addition, the impact of uncertainty associated with single parameter values on the cost-effectiveness was assessed by analysis of covariance methods. ${ }^{28}$ The potential value of collecting further evidence on uncertain parameters was quantified using expected value of perfect information (EVPI) analysis.

Scenario analysis. We performed a deterministic one-way sensitivity analysis to assess the impact of systematically varying one single input parameter at a time across wider intervals. Furthermore, we performed extensive scenario analysis assessing the impact of different assumptions on testing uptake,

Table 1 Discounted costs and effects of prevention in mutation carriers

\begin{tabular}{|c|c|c|c|c|c|c|c|}
\hline & $\begin{array}{l}\text { Costs } \\
\text { of CRC }\end{array}$ & $\begin{array}{l}\text { Costs of } \\
\text { colonoscopic } \\
\text { prevention }\end{array}$ & $\begin{array}{c}\text { Costs of } \\
\text { aspirin } \\
\text { prevention }\end{array}$ & $\begin{array}{l}\text { Total } \\
\text { costs }\end{array}$ & $\begin{array}{l}\text { Discounted } \\
\text { LYs men }\end{array}$ & $\begin{array}{l}\text { Discounted } \\
\text { LYs women }\end{array}$ & $\begin{array}{l}\text { Discounted } \\
\text { LYs average }\end{array}$ \\
\hline Standard prevention & 5,023 & 245 & 0 & 5,268 & 20.26 & 21.77 & 20.90 \\
\hline Annual colonoscopy and aspirin prevention & 1,561 & 4,300 & 596 & 6,460 & 20.75 & 22.35 & 21.42 \\
\hline Increment to standard prevention & $-3,462$ & 4,055 & 596 & 1,192 & 0.49 & 0.58 & 0.52 \\
\hline
\end{tabular}

CRC, colorectal cancer; LY, life-year. 
performance of revised Bethesda criteria, aspirin prophylaxis, screening intervals, decreasing testing costs, and discount rates.

\section{RESULTS}

\section{Costs per mutation detected}

Assuming an LS prevalence of 3\%, 2,082 of 69,400 newly diagnosed CRC cases are affected with LS. In the base case analysis, the number of detected mutations among CRC cases ranges from 383 in strategies A-5 and A-6 to 1,646 in strategy 7.

Overall testing costs in index patients range between $€ 11,308,328$ in strategy B-2 and $€ 257,044,078$ in the direct sequencing strategy (strategy 7). Strategy B-2 has the lowest cost per index mutation detected, resulting in a cost of $€ 17,935$ per mutation detected.

\section{Effects of LS screening}

The expected undiscounted number of LYG through prevention in LS carriers is 1.61 years for men and 1.98 years for women when prevention is started at age 25 years, and decreases with the patient's age at the time prevention is started. Women gain more life-years through prevention than men because of their overall higher life expectancy. The average gain by increased prevention is 1.34 LYG (1.20 for men and 1.52 for women; LYG discounted at $3 \%$ are 0.49 and 0.58 , respectively).

Mean costs and effects of prevention for a relative with LS are shown in Table 1. Total costs of care with increased prevention for relatives aged 25 years and older outweigh the cost associated with standard prevention in undetected LS carriers by $€ 1,192$ per person. The major part of the costs of increased prevention is due to colonoscopic surveillance (€4,300 of $€ 4,896)$.

\section{Cost-effectiveness of LS screening}

All screening strategies reduce cancer incidence and death and yield more life-years than the no-screening strategy. The vast majority of screening strategies are dominated (meaning that they are more costly and less effective than alternative strategies considered) or subject to extended dominance (meaning that they are dominated by a combination of two or more alternative strategies considered). Table 2 displays the costs, effects, and incremental cost-effectiveness ratios (ICERs) as compared with the next most cost-effective strategy of the remaining screening strategies. The ICERs range from $€ 77,268$ per LYG in strategy B-2 to $€ 4,188,036$ per LYG in strategy 7.

Table 2 Cost-effectiveness without dominated screening alternatives (as compared with the next most cost-effective strategy)

\begin{tabular}{llcrrrr} 
Strategy & Total costs & Total LYs & $\begin{array}{c}\text { Incremental } \\
\text { costs }\end{array}$ & $\begin{array}{c}\text { Incremental } \\
\text { LYs }\end{array}$ & $\begin{array}{c}\text { Incremental } \\
\text { costs per LY } \\
\text { gained }\end{array}$ \\
\hline 0 & No screening & $218,581,280$ & $5,703,154$ & - & - & - \\
B-2 & Counseling including Bethesda, IHC, BRAF, sequencing & $242,028,209$ & $5,703,458$ & $23,446,929$ & 303 & 77,268 \\
2 & Counseling, IHC, BRAF, sequencing & $252,442,654$ & $5,703,499$ & $10,414,444$ & 41 & 253,258 \\
7 & Counseling, direct sequencing & $476,882,146$ & $5,703,552$ & $224,439,493$ & 54 \\
\hline
\end{tabular}

BRAF, BRAF V600E mutation testing; IHC, immunohistochemistry; LY, life-year.

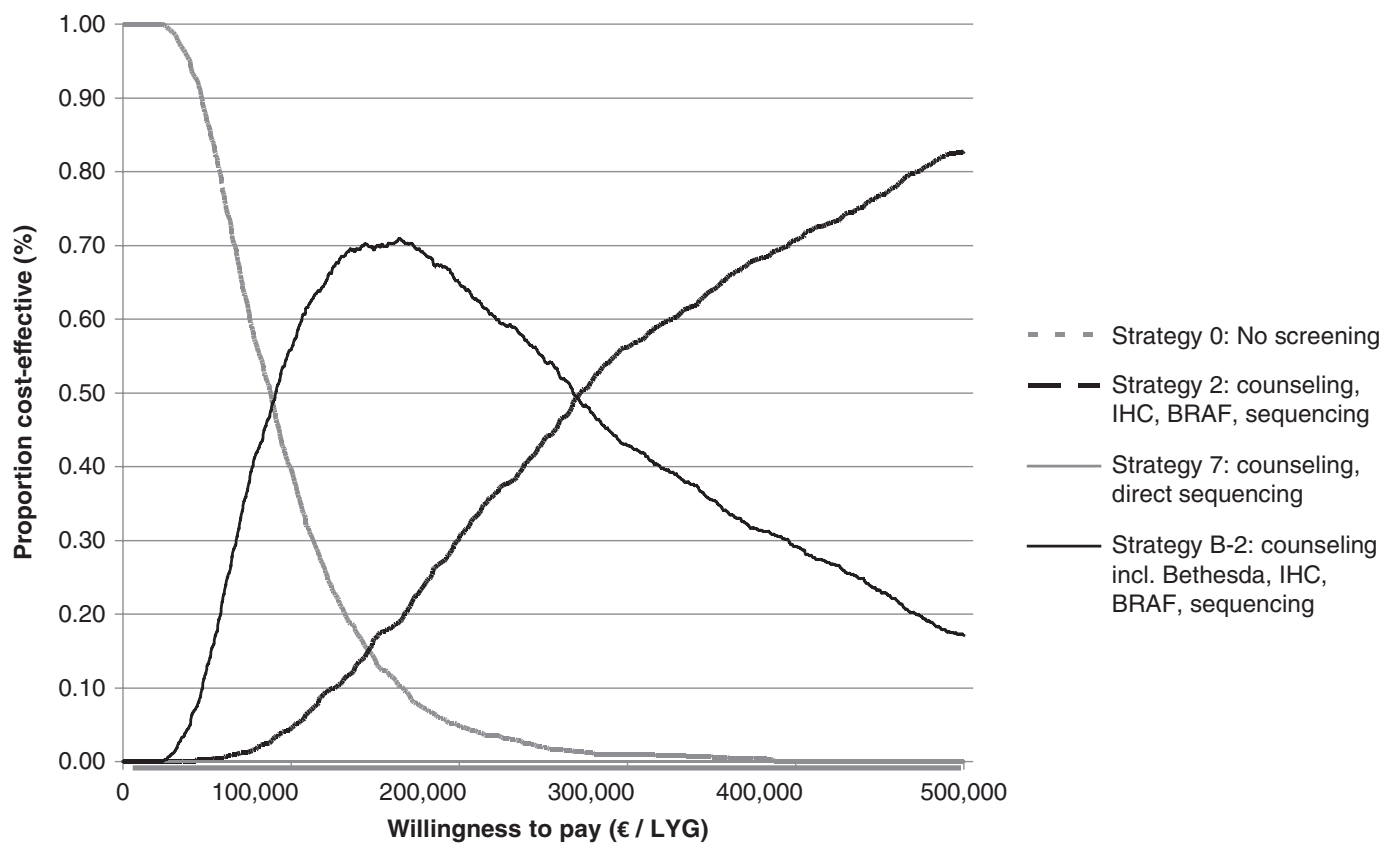

Figure 3 Cost-effectiveness acceptability curves. 


\section{Probabilistic sensitivity analysis}

Figure 3 displays the cost-effectiveness acceptability curve for the nondominated screening strategies. At a threshold of $€ 50,000$ per LYG used for illustration, the no-screening strategy has a probability of $87 \%$ of being considered cost-effective. At the same threshold, strategy B-2 is the optimal strategy in 13\% of iterations.

A summary of the analysis of covariance results for strategy B-2 applied separately to LYG and incremental costs is presented in Supplementary Material S2 online. This analysis suggests that stage distribution of CRC in LS carriers who do not adhere to colonoscopic surveillance and the prevalence of LS are the most important factors in explaining variation in the overall effects. In Germany, the ambulatory care fee schedule includes a floating evaluation factor. Uncertainty about the parameter distribution of this value has the largest impact on the overall distribution of costs.

The EVPI is plotted in Supplementary Material S3 online for willingness to pay thresholds between $€ 0$ and $€ 500,000$ per LYG and a time horizon of 5 years. The EVPI indicates that further research to inform decisions could be worthwhile. At a threshold of $€ 50,000$ and a time horizon of 5 years, the EVPI amounts to $€ 3,360,954$. Extending the analysis to an infinite time horizon increases the EVPI to $€ 23,750,152$.

\section{Scenario analyses}

To identify important drivers of cost-effectiveness of LS screening, a number of scenarios were explored that are fully reported in Supplementary Material S4 online. Variables concerning uptake of testing appear to be critical drivers of cost-effectiveness. Increasing the uptake rate among FDRs to 52\% (ref. 32) changes the ICER of strategy B-2 to $€ 45,169$ per LYG as compared with no screening. Assuming an ideal uptake of $100 \%$ in index patients and FDRs results in an ICER of $€ 24,979$ per LYG.

Omitting aspirin chemoprevention from the model is associated with only a small reduction in health benefits. As compared with the baseline analysis, average remaining life expectancy of LS carriers decreases by only 0.01 years. Moreover, aspirin prophylaxis has only a small influence on the cost-effectiveness of LS screening. If aspirin is omitted from the model, the ICERs increase slightly, with, for example, strategy B-2 resulting in $€ 79,812$ per LYG.

Supplementary Material S5 online also reports the results of a deterministic one-way sensitivity analysis systematically varying all input parameters across wider intervals. The number of relatives per index patient has the highest impact on model results. Strategies become more cost-effective as more relatives are tested. The prevalence of LS is another influential variable. As the prevalence decreases, calculated cost per LYG increases.

\section{DISCUSSION}

In this study, we evaluated the effectiveness and cost-effectiveness of LS screening as a potential strategy for individualized CRC prevention. The cost-effectiveness of the included strategies ranges between $€ 77,268$ per LYG in strategy B-2 and $€ 4,188,036$ per LYG in strategy 7.

\section{Implication of this study}

LS screening recommendations in Germany foresee that patients who seek genetic counseling will be assessed by the revised Bethesda criteria followed by either an MSI analysis (strategy B-6) or a sequential IHC and MSI analysis (strategy B-4). ${ }^{5}$ In our analysis, both strategies were dominated. This means that other strategies considered in this analysis are both less expensive and more effective in detecting LS carriers. To improve the health benefits derived from the available budget, a revised version of the German recommendations for LS screening might take these results into account and promote the increased use of more cost-effective strategies involving IHC and $B R A F$ testing in newly diagnosed CRC patients.

To our knowledge this is the first cost-effectiveness analysis that accounts for the presumably favorable effect of aspirin chemoprevention as an "add-on" to colonoscopic surveillance in LS carriers. According to our results, aspirin chemoprevention makes only a small contribution to CRC risk reduction. In mutation carriers who adhere to recommended colonoscopic screening, aspirin use provides relatively low additional health effects. Despite the additional costs associated with aspirin use and the treatments of aspirin-related complications, aspirin chemoprevention slightly improves the cost-effectiveness of LS screening. However, we analyzed the role of aspirin only when it is used as an add-on and not its impacts when it is used instead of colonoscopic surveillance. Because colonoscopic surveillance is unpleasant in preparation, time-consuming, and not without risk, some LS carriers might prefer solely aspirin prevention despite the uncertainty surrounding its effects. Further studies that assess the effectiveness and cost-effectiveness of LS screening in a population solely adhering to aspirin chemoprevention would thus be desirable. Given that the effectiveness of aspirin in reducing CRC is uncertain and given that it is associated with additional adverse effects, CRC prophylaxis should take individual patient preferences into account.

Deterministic sensitivity analysis demonstrated that variables concerning uptake of testing appear to be critical drivers of cost-effectiveness. If all index patients and their FDRs were tested for LS, the calculated ICER could be decreased to $€ 24,979$ per LYG. Although this is an unrealistic scenario, it illustrates a key determinant of cost-effectiveness. Because costs of testing index patients occur regardless of how many FDRs are tested subsequently, low uptake by index cases is relatively less important than that by FDRs. This indicates that further research into factors that could enhance FDRs' willingness to undergo testing would be desirable. If tested index cases are reluctant to share relevant genetic information with their family members, relatives cannot benefit from screening and intervention. The cost-effectiveness of screening could be increased if physicians routinely report genetic information to family members at increased risk. However, for such a strategy, legal and ethical 
restrictions regarding the protection of confidential patient information need to be considered carefully.

\section{Comparison with other studies}

The results from this study differ substantially from the favorable results of previous cost-effectiveness models used to evaluate LS screening in patients with newly diagnosed CRC. ${ }^{12-19}$ Based on current screening recommendations in Germany, we assume that mutation-positive patients undergo colonoscopy once per year, whereas previous studies assumed screening intervals of 2 or even 3 years. ${ }^{12,14-17}$ The sensitivity analysis presented in Supplementary Material S4 online shows, however, that the closed-meshed surveillance foreseen by the German guidelines does not explain the differences in the model results. Rather, this might be explained by the following factors.

First, we assume that only a small proportion of relatives are tested for LS. This is due to the low probability of relatives being interested in the screening program. Other studies assume higher uptake ranging between 52 and $100 \%$ for FDRs. ${ }^{13,14,17,18}$ In Germany, however, experience has shown that the number of relatives who are tested for LS and thus can benefit from screening is likely to be smaller, which tends to increase the costs per LYG.

Second, other studies also assume more FDRs per index patient, with the studies by Ladabaum et al. ${ }^{13}$ and Wang et al. ${ }^{18}$ assuming up to eight FDRs per index patient, all of whom are 25 years of age when prevention starts. In this study, we assume less than four FDRs per index patient based on German evidence. ${ }^{25}$ This is because family size in Germany tends to be smaller than in the United States. Results from deterministic and probabilistic sensitivity analysis have shown that the number of relatives tested is a critical driver of cost-effectiveness. Also, assumptions made by Ladabaum et al..$^{13}$ and Wang et al..$^{18}$ relating to relatives' age are likely to improve cost-effectiveness as health gains decrease with the patient's age at the time prevention is started.

The differences in life expectancy between LS carriers that adhere to increased prevention and those that do not adhere to increased prevention were slightly higher in previous studies. ${ }^{13,14,18}$ This can be explained by different assumptions relating to cancer stage distributions and cancer risks in LS patients. Mvundura et al. ${ }^{14}$ assumed ultimately less favorable stage distributions and cancer stage-related survival for LS patients as compared with this study. This might have overestimated the degree of benefit derived from increased prevention. Also, the study by Mvundura et al. ${ }^{14}$ assumed better cancer stage-related survival for those LS patients adhering to colonoscopic prevention as compared with those who do not adhere to prevention. The studies by Ladabaum et al. ${ }^{13}$ and Wang et al..$^{18}$ assumed ultimate high CRC risk based on earlier studies assessing risks in mutation carriers. However, there is evidence from more recent studies that CRC risks are lower than previously reported. ${ }^{2,4}$ Again, this is likely to underestimate the costs per LYG of CRC prevention.

\section{Limitations}

Our study has several limitations. First, we did not model the risk of other forms of cancer. In particular, women face an increased risk for gynecological cancers (e.g., endometrial or ovarian cancer). Although the German guidelines foresee gynecological surveillance, the efficiency of such examinations is still uncertain..$^{33}$ To our knowledge, the studies by Ladabaum et al. ${ }^{13}$ and Dinh et al. ${ }^{19}$ are the only cost-effectiveness studies addressing the critical issue of screening for gynecological cancers. Due to the uncertain benefit derived from such screening, the studies assume that gynecological surveillance results in additional costs without comprising any benefits.

We did not assume different CRC risks and prevention intervals based on the MMR gene involved. MSH6 carriers have been found to be at lower risk for CRC with a higher age of tumor onset as compared with $\mathrm{MLH1}$ and $\mathrm{MSH} 2$ carriers. ${ }^{4}$ This probably means that no general prevention protocol is suitable for all MMR mutation carriers. Also, we did not model CRC risk associated with the EPCAM gene, another gene that has recently been found to impact LS. ${ }^{34}$

We did assume that mutation carriers adhering to regular colonoscopy also adhere to aspirin prevention. This might have biased our results for or against screening. However, reported adherence rates in clinical trials evaluating aspirin for the prevention of colorectal adenomas are high and range between 76 and $92 \% .{ }^{35}$ In mutation carriers, aspirin compliance might even be higher because these patients might be aware of their predisposition. Also, like other studies, ${ }^{12-16,18}$ ours did not model ongoing adherence rates for CRC prevention. If relatives decide to stop prevention, this would reduce LYG and calculated ICERs might be underestimated. However, studies have found that lifetime compliance is high $^{14}$ and that $95 \%$ of mutation-positive relatives would consider lifetime surveillance. ${ }^{36}$

Furthermore, risks for complications of increased prevention in mutation carriers might be underestimated. Our estimates concerning the risk of complications derived from colonoscopy are based on data from the German screening program..$^{37}$ This program is, however, aimed at the general population aged 55 years and older. It might be that estimates are too optimistic and that, in reality, risks of complications are higher in the LS group. Risk of bleeding after aspirin intake was taken from a recent meta-analysis assessing the effect of aspirin on vascular outcomes. ${ }^{38}$ However, patients analyzed in this study in general received a lower aspirin dose than assumed in the current study. Patients taking high-dose aspirin might have higher risks for adverse events. Because reported risks are low, we assume a zero risk of death from colonoscopy and aspirin prevention. Between 2003 and 2008, only five fatalities from colonoscopy were reported, with 1 million colonoscopies performed in Germany. Death from gastrointestinal bleeding is also rare. A review of aspirin prevention for CRC reported only one death associated with gastrointestinal bleeding. ${ }^{39}$ We also did not model increased risk 
for hemorrhagic stroke in patients taking aspirin. However, any increase in risk for hemorrhagic stroke might be compensated by a risk reduction for ischemic stroke. ${ }^{40}$

We did not calculate outcomes in terms of quality-adjusted life-years. We would expect that by including quality-adjusted life-year weights, the cost-effectiveness of LS screening would become less favorable. Mvundura et al. ${ }^{14}$ showed that including quality-adjusted life-years raises the costs per health outcome, reflecting the fact that most people are not in perfect health. This result is confirmed by the study of Wang et al., ${ }^{18}$ which incorporated preference data from their own quality-of-life study.

\section{Conclusion}

In this study we evaluated the cost-effectiveness of LS screening. Results have shown that LS screening provides clinical benefit but at a high cost. The most cost-effective strategy involves family-history assessment with the revised Bethesda criteria, followed by IHC testing, BRAF testing, and genetic sequencing, with an ICER of $€ 77.268$ per LYG. A revised version of the German recommendations for CRC might wish to take these results into account and promote IHC and subsequent BRAF testing instead of MSI testing. To improve the cost-effectiveness of LS screening, further research should address factors that could enhance FDRs' willingness to undergo testing.

To our knowledge, this is the first economic evaluation that also considers aspirin prophylaxis as an "add-on" to intensified colonoscopic screening for CRC prevention in LS patients. In our analysis, aspirin chemoprevention provides comparatively low additional health effects and improves the cost-effectiveness of screening only slightly. Given uncertainty surrounding its long-term effects and side effects, the economic case for recommending chemoprevention as an adjunct to colonoscopy is rather weak. Physicians thus might wish to consider other factors such as individual patient preferences when prescribing chemoprevention.

\section{SUPPLEMENTARY MATERIAL}

Supplementary material is linked to the online version of the paper at http://www.nature.com/gim

\section{ACKNOWLEDGMENTS}

This research was supported by a grant funded by the German Federal Ministry of Education and Research (BMBF) within the context of the "ethical, legal, and social aspects of modern life sciences and biotechnology" ELSA project (grant 01GP1006A-C). We are grateful to Scott Grosse and Verena Steinke-Lange for their valuable comments regarding earlier versions of the manuscript.

\section{DISCLOSURE}

F.S., E.H.F., E.M., V.H., C.G.J., and W.R. declare no conflict of interest. B.S. declares no conflict of interest while the work was conducted at Helmholtz Zentrum München. Since July 2014, B.S. is an employee of AMGEN Europe $(\mathrm{GmbH})$, who also provides cancer drugs.

\section{REFERENCES}

1. RKI. Beiträge zur Gesundheitsberichterstattung des Bundes: Krebs in Deutschland 2007/2008. http://www.rki.de/DE/Content/ Gesundheitsmonitoring/Gesundheitsberichterstattung/GBEDownloadsB/ KID2012.html. Accessed 2 December 2013.

2. Palomaki GE, McClain MR, Melillo S, Hampel HL, Thibodeau SN. EGAPP supplementary evidence review: DNA testing strategies aimed at reducing morbidity and mortality from Lynch syndrome. Genet Med 2009;11:42-65.

3. Laghi L, Malesci A. Microsatellite instability and therapeutic consequences in colorectal cancer. Dig Dis 2012;30:304-309.

4. Bonadona V, Bonaïti B, Olschwang S, et al.; French Cancer Genetics Network. Cancer risks associated with germline mutations in $\mathrm{MLH} 1, \mathrm{MSH} 2$, and MSH6 genes in Lynch syndrome. JAMA 2011;305:2304-2310.

5. Leitlinienprogramm Onkologie (Deutsche Krebsgesellschaft, Deutsche Krebshilfe, AWMF): S3-Leitlinie Kolorektales Karzinom, Langversion 1.1, 2014, AWMF Registrierungsnummer: 021-0070L. http://leitlinienprogrammonkologie.de/Kolorektales-Karzinom.62.0.html. Accessed 29 July 2014.

6. Engel C, Rahner N, Schulmann K, et al.; German HNPCC Consortium. Efficacy of annual colonoscopic surveillance in individuals with hereditary nonpolyposis colorectal cancer. Clin Gastroenterol Hepatol 2010;8:174-182.

7. Stupart DA, Goldberg PA, Algar U, Ramesar R. Cancer risk in a cohort of subjects carrying a single mismatch repair gene mutation. Fam Cancer 2009;8:519-523.

8. Burn J, Gerdes AM, Macrae F, et al.; CAPP2 Investigators. Long-term effect of aspirin on cancer risk in carriers of hereditary colorectal cancer: an analysis from the CAPP2 randomised controlled trial. Lancet 2011;378:2081-2087.

9. Tan YY, McGaughran J, Ferguson K, et al.; ANECS Group. Improving identification of Lynch syndrome patients: a comparison of research data with clinical records. Int J Cancer 2013;132:2876-2883.

10. EGAPP Working Group. Recommendations from the EGAPP Working Group: genetic testing strategies in newly diagnosed individuals with colorectal cancer aimed at reducing morbidity and mortality from Lynch syndrome in relatives. Genet Med 2009;11:35-41.

11. NCCN Clinical Practice Guidelines in Oncology (NCCN Guidelines). Genetic/ familial high-risk assessment: colorectal. http://www.nccn.org/professionals/ physician_gls/f_guidelines.asp. Accessed 17 September 2014.

12. Kievit W, de Bruin JH, Adang EM, et al. Cost effectiveness of a new strategy to identify HNPCC patients. Gut 2005;54:97-102.

13. Ladabaum U, Wang G, Terdiman J, et al. Strategies to identify the Lynch syndrome among patients with colorectal cancer: a cost-effectiveness analysis. Ann Intern Med 2011;155:69-79.

14. Mvundura M, Grosse SD, Hampel H, Palomaki GE. The cost-effectiveness of genetic testing strategies for Lynch syndrome among newly diagnosed patients with colorectal cancer. Genet Med 2010;12:93-104.

15. Ramsey SD, Burke W, Clarke L. An economic viewpoint on alternative strategies for identifying persons with hereditary nonpolyposis colorectal cancer. Genet Med 2003;5:353-363.

16. Ramsey SD, Clarke L, Etzioni R, Higashi M, Berry K, Urban N. Costeffectiveness of microsatellite instability screening as a method for detecting hereditary nonpolyposis colorectal cancer. Ann Intern Med 2001;135(8 Pt 1):577-588.

17. Wang VW, Koh PK, Chow WL, Lim JF. Predictive genetic testing of first degree relatives of mutation carriers is a cost-effective strategy in preventing hereditary non-polyposis colorectal cancer in Singapore. Fam Cancer 2012;11:279-289.

18. Wang G, Kuppermann M, Kim B, Phillips KA, Ladabaum U. Influence of patient preferences on the cost-effectiveness of screening for Lynch syndrome. J Oncol Pract 2012;8(3 suppl):e24s-e30s.

19. Dinh TA, Rosner BI, Atwood JC, et al. Health benefits and cost-effectiveness of primary genetic screening for Lynch syndrome in the general population. Cancer Prev Res (Phila) 2011;4:9-22.

20. Hagen A, Hessabi HK, Gorenoi V, Schönermark MP. [Cost-effectiveness evaluation of predictive molecular diagnostics using the example of hereditary non-polyposis colorectal cancer (HNPCC)]. Gesundheitswesen 2008;70:18-27.

21. Busse R, Blümel M. Germany: health system review. Health Syst Transit, 2014;16:1-296. http://www.euro.who.int/en/about-us/partners/observatory/ health-systems-in-transition-hit-series/countries-and-subregions/germanyhit-2014. Accessed 9 October 2014.

22. Vasen HF, Watson P, Mecklin JP, Lynch HT. New clinical criteria for hereditary nonpolyposis colorectal cancer (HNPCC, Lynch syndrome) proposed by the International Collaborative group on HNPCC. Gastroenterology 1999;116:1453-1456. 
23. Umar A, Boland CR, Terdiman JP, et al. Revised Bethesda Guidelines for hereditary nonpolyposis colorectal cancer (Lynch syndrome) and microsatellite instability. J Natl Cancer Inst 2004;96:261-268.

24. Hampel H, Frankel WL, Martin E, et al. Feasibility of screening for Lynch syndrome among patients with colorectal cancer. J Clin Oncol 2008;26:57835788.

25. Schneider R, Rümmele P, Dechant S, Hofstädter F, Lorenz W, Fürst A. [Familia non-polyposis colorectal carcinoma (Lynch syndrome) in Germany - analysis of information, advisory service and family screening]. Dtsch Med Wochenschr 2011;136:17-22.

26. Ramsoekh D, van Leerdam ME, Tops CM, et al. The use of genetic testing in hereditary colorectal cancer syndromes: genetic testing in HNPCC, (A)FAP and MAP. Clin Genet 2007:72:562-567.

27. Moreira L, Balaguer F, Lindor N, et al.; EPICOLON Consortium. Identification of Lynch syndrome among patients with colorectal cancer. JAMA 2012;308:15551565.

28. Briggs A, Claxton C, Sculpher M. Decision Modelling for Health Economic Evaluation. Oxford University Press: Oxford, UK, 2006

29. Robert-Koch-Institute. Centre for Cancer Registry Data. http://www.rki.de/ EN/Content/Health_Monitoring/Cancer_Registry/cancer_registry_node.html. Accessed 12 March 2012.

30. Popat $\mathrm{S}$, Hubner R, Houlston RS. Systematic review of microsatellite instability and colorectal cancer prognosis. J Clin Onco/ 2005;23:609-618.

31. Institut für Entgeldsysteme im Krankenhaus. http://www.g-drg.de/cms Accessed 30 July 2013.

32. Sharaf RN, Myer P, Stave CD, Diamond LC, Ladabaum U. Uptake of genetic testing by relatives of Lynch syndrome probands: a systematic review. Clin Gastroenterol Hepato/ 2013;11:1093-1100.
33. Vasen HF, Blanco I, Aktan-Collan K, et al.; Mallorca group. Revised guidelines for the clinical management of Lynch syndrome (HNPCC): recommendations by a group of European experts. Gut 2013;62:812-823.

34. Kuiper RP, Vissers LE, Venkatachalam R, et al. Recurrence and variability of germline EPCAM deletions in Lynch syndrome. Hum Mutat 2011;32:407-414.

35. Cole BF, Logan RF, Halabi S, et al. Aspirin for the chemoprevention of colorectal adenomas: meta-analysis of the randomized trials. J Natl Cancer Inst 2009;101:256-266

36. Lynch HT, Watson P, Shaw TG, et al. Clinical impact of molecular genetic diagnosis, genetic counseling, and management of hereditary cancer. Part II: hereditary nonpolyposis colorectal carcinoma as a model. Cancer 1999:86(11 suppl):2457-2463.

37. Zentralinstitut für die kassenärztliche Versorgung in der Bundesrepublik Deutschland. Projekt wissenschaftliche Begleitung von FrüherkennungsKoloskopien in Deutschland. 2008.http://www.google.com/url?sa=t\&rct=j\&q= \&esrc $=s \&$ source $=$ web \&cd $=1 \&$ ved $=0 C B 8 Q F j A A \& u r l=h t t p \% 3 A \% 2 F \% 2 F w w w$. aerzteblatt.de\%2Fdownload\%2Ffiles\%2F2010\%2F03\%2Fdown144345.pdf \&ei=GKqFVLfrFoXjO73KgMgF\&usg=AFQjCNFBWjxlepvkSMdxpfbOZ7JfjwN5g\&bvm=bv.80642063,d.ZWU. Accessed 1 December 2013.

38. Raju N, Sobieraj-Teague M, Hirsh J, O'Donnell M, Eikelboom J. Effect of aspirin on mortality in the primary prevention of cardiovascular disease. Am J Med 2011:124:621-629.

39. Dubé C, Rostom A, Lewin G, et al.; U.S. Preventive Services Task Force. The use of aspirin for primary prevention of colorectal cancer: a systematic review prepared for the U.S. Preventive Services Task Force. Ann Intern Med 2007:146:365-375

40. Thorat MA, Cuzick J. Role of aspirin in cancer prevention. Curr Oncol Rep 2013:15:533-540. 